\title{
Healthy weight maintenance strategy in early childhood: the views of Black African migrant parents and health visitors
}

\section{Abstract}

In Europe and the US, childhood obesity is found to be higher in migrant children from Black African communities and other visible minority ethnic groups. However, very little is known about the factors that contribute to the significant rates of obesity in these groups.

The material for this paper is drawn from a community-based qualitative study that examined the socio-cultural, familial, and environmental factors that either facilitate or hinder healthy weight in Black African children during early childhood. The participants for the study were Black African parents $(n=30)$ and health visitors $(n=32)$, residing and working in the East Midlands, UK. The participants were purposively selected according to an inclusion/exclusion criterion and invited to participate in seven focus groups (FG-7) conducted for parents (FG-4) and health visitors (FG-3) at a time and place convenient to the participants, between March and June 2018.

The focus groups examined a number of issues, including the participants' views on the barriers and facilitators to achieving and maintaining a healthy weight. Thematic analysis was used to identify themes within the data. Although participants were knowledgeable about the need for a healthy weight in early childhood, the parents discussed how immigration status and experiences of discrimination had an impact on their children's diet and wellbeing, also discussing how structural factors influenced the decisions they made regarding healthy weight in early childhood. While the health visitors in general felt they did not have the skills to advise Black African parents about the cultural influences on diet during early childhood, findings highlighted a need for a system-based approach in meeting the nutritional needs of Black African children. 
This study concludes by suggesting the need for broad social-environment and economic changes that address factors such as migration status, deprivation, and discrimination, coupled with culturally specific healthy diet messages.

\section{What is known about this topic}

- Globally, more than 41 million children under the age of five are either overweight or obese.

- Obesity in childhood is a strong predictor of obesity in adulthood.

- Childhood obesity increases the risk of a number of serious health conditions later in life, which have serious implications on the sustainability of health and social care systems.

\section{What this paper adds}

- Uncertainty in immigration status and socio-economic disadvantages, including discrimination, were found to constrain Black African parents' ability to promote healthy weight maintenance in early childhood.

- There is a need for health and social care practitioners to adopt a partnership model and to develop interventions that could address factors such as migration status, deprivation, and social discrimination in order to promote healthy weight in early childhood for migrant children.

Key words: black African parents, Health Visitors, early-childhood, focus groups, obesity, weight maintenance 


\section{Introduction}

Worldwide, more than 41 million children under the age of five are either overweight or obese (WHO, 2016). Specifically, the UK, Europe, and the US are battling a childhood obesity epidemic with nearly a third of children either overweight or obese, and with them staying obese for a longer period of time (HC, 2018). Obesity in childhood is a strong predictor of obesity in adulthood, as well as predisposing chronic illnesses such as diabetes, cancer, cardiovascular diseases, and mental illness later in life (Simmonds et al., 2015). Consequently, the increase in childhood obesity suggests that this generation of children are likely to have a lower life expectancy than their parents. Unfortunately, obesity prevalence in England is found to be higher in children who are from Black and minority ethnic (BME) groups compared with children from the White British population (Gatineau and Mathrani, 2011; HSCIC, 2018; PHE, 2019; NHSHSC, 2013). Findings from the National Child Measurement Programme (HSCIC, 2018) indicate that, by the time children are 4-5-years old, obesity prevalence is highest in the Black African groups.

Increasing childhood obesity leads to major health and social care concerns, as well as serious implications on the sustainability of a country's health and social care systems (Dawe, 2015). For instance, the UK National Health Service (NHS) spent $£ 16$ billion a year on overweight and obesityrelated illnesses (Hughes, 2016), which is more than the government spent on police, fire services, and the judicial system combined. Consequently, if obesity prevention is not prioritised, the associated financial costs for families and communities will continue to rise, placing a continuing burden on the UK and European health and social care systems.

Given the rising prevalence of obesity in children, it is anticipated that obesity levels in BME children, both in UK and globally, will increase 
dramatically, with these increases affecting their health and wellbeing later on in life. Hence, tackling childhood obesity by focusing on the early years has become a key priority. However, to date, there is a dearth of knowledge that could inform the development of effective strategies to prevent childhood obesity in BME children in the UK and Europe. Consequently, there is an urgent need to examine the predisposing factors of obesity in visible minority communities, as well as their beliefs, perceptions, and experiences of childhood obesity and healthy weight maintenance. Improving children's health would lead to better health outcomes later in life, which could also contribute towards the reduction of inequalities in health.

As families are the best paradigm for healthy weight in early childhood (Kvaakik, 2003; Guo and Chimlea, 1999), advocates of healthy weight maintenance programmes in early childhood have argued that parents should be at the centre of such programmes. Involving parental contribution when examining the risk of obesity in early childhood is likely to inform prevention programmes targeted to promote healthy weight maintenance during this period (Kvaakik, 2003; Maynard et al., 2009). Parents can also be role models to their children, with their support subsequently making weight loss easier for the children (Kelishadi \& AziziSoleiman, 2014). Therefore, developing healthy weight strategies with parents in early childhood is critical (Golley et al., 2007; Cheng, 2012). Such strategies, however, should be multifaceted and should actively involve all stakeholders working with families in order to promote healthy weight in early childhood (Blüher, 2019).

Given that health visitors (HVs) are specialist public health nurses, who work directly with children and their parents/carers, they are best placed to promote healthy weight in early childhood ( $\mathrm{DH}, 2012)$. The role of HVs 
is underlined by The Healthy Child Programme (PHE, 2018), which recognises the importance of promoting a healthy childhood, including the promotion of healthy weight. Critically, HVs are in contact with all children (at stipulated intervals) from before birth up to five years of age, and during this time they offer caregivers various advice, including information on: healthy growth, infant feeding, sleep patterns, and monitoring child development (DH, 2009). Consequently, HVs should have the necessary skills to work with caregivers from different ethnic, cultural, and socialeconomic backgrounds in order to respond with confidence to issues they may experience when child rearing (for a discussion of the role of HVs see Baldwin 2012). However, there is a gap in research studies regarding the experiences of HVs working with Black African communities in order to promote healthy weight maintenance in early childhood. This project, therefore, also investigated the experiences of HVs working with the Black African community to promote healthy weight maintenance.

\section{Methods}

The central aim of this project was to examine the factors that facilitate or hinder healthy weight in Black African children during early childhood. Ethical approval for the study was granted by the authors institution (HLS FREC Ref: 2065) and the NHS Health Research Authority (Ref: 18/HRA/0451). Sampling was designed to recruit Black African parents and HVs from the East Midlands, UK. For the Black African parents, they were required to have a child or children between the ages of six months and five years living at home, as well as the ability to communicate in English and consent to participate in a focus group. The focus was on Black African migrants who have been residing in the UK for $>5$ years, as (noted previously) BME children in England have the highest rates of obesity. The researcher, who herself is of Black African descent heritage, applied the principles of purposive sampling (Bryman, 2016) to identify the parents, 
recruiting them through community and spiritual organisations that catered for BME communities (see authors reflexive account on researching with Black African families, Ochieng 2010). The ethnic origin of the parents was identified using the UK Census categories (Census, 2011). A total of 30 Black African parents ( 15 women and 15 men) between the ages of 24-55 years took part in the focus groups. The HVs (n-32) were all female and were all actively employed in the East Midlands. Four of them were South Asian, two were of Black African descent heritage, one was dual heritage (Black African heritage and White British), and twenty-five were White British.

Four focus groups ( $F G$ ) for parents ( $F G$ female $n=2$ and FG male $n=2$ ), and three FG for the HVs were conducted during the months of March - June 2018, with the sites for the FGs being agreed on beforehand with the participants. These included: NHS settings, places of worship, and community centres. Each participant received written and oral explanations of the project, they were assured of confidentiality, and informed consent was obtained. There were $7-12$ participants for each FG, the main advantage of this sample size being that it allowed an in-depth focus on the experiences of the participants. The gender-specific focus groups were designed to better facilitate and fully capture the views among women and men (Dyck et al., 2019). This was particularly important considering how little data is available on the subject in the UK.

All the focus groups were facilitated by the researcher using an FG schedule, to examine: the participants' descriptions of healthy weight in early childhood, barriers to healthy weight, factors facilitating and inhibiting healthy weight maintenance, and strategies that will support parents to enable healthy weight maintenance in early childhood. At the beginning of each FG meeting, participants were reminded of the purpose of the project, 
as well as any ethical considerations such as anonymity and confidentiality. All parents were given a voucher to overcome the potential costs involved with taking part in the FG, such as travelling costs and money for childcare. At the end of each FG, the researcher delivered a verbal summary of the key issues that had emerged and provided participants with the opportunity to raise any other related issues. The FGs lasted between 50-90 minutes, and all discussions were recorded using a digital voice recorder and transcribed accordingly.

Thematic analysis (Bryman, 2016) was used to identify themes within the data, and this process was facilitated by the use of a qualitative software package - Nvivo 11 (Lacey and Loft, 2009). The researcher had already developed a number of general themes through ongoing reflection during the focus group process, which were used for the initial coding. In analysing the findings from the different parents' groups, the issues that emerged were broadly similar irrespective of the participants' number of years in the UK and country of origin, therefore their findings are presented as a part of a whole. However, there were some differences based on gender, which have been highlighted. The interim findings were also discussed with Black African parents and HVs at different stages of the focus group process. This ensured that the developing categories were refined and informed by the participants and minimise the influence of the researchers' own interpretations and this resulted in three broad categories: Concept of healthy weight and diet in childhood, Challenges to healthy weight maintenance in early childhood and an integrated approach to promote healthy weight in childhood.

\section{Results}

Concept of healthy weight and diet in childhood 
The concept of healthy weight and diet was complex and multi-dimensional, involving interactions between social, cultural, familial, economic, and environmental factors.

I'm not really concerned about my son's weight, I don't look at his weight but I look at, like, for example: does he get sick a lot or is he fine? (Parents FG -1)

Knowing that a child that young is a healthy weight is difficult. As far as I'm concerned my child looks, like, not overweight and not very thin, medium sized. (Parents FG-2)

Though all the parents demonstrated substantial knowledge and understood the description of a healthy diet, they were influenced by a number of factors, including:

African heritage diet and weaning practices

I am an African and I think our food [referring to African heritage foods] is very important, and for me, I want my children to eat our food. As a family I mostly try to cook our food although it can take a lot of time to prepare, but that's our heritage and I want my children to know this diet. (Parents'FG3)

I also think our food should be recognised in healthy food messages for children. It is important to us. (Parents' FG-4)

For Black African parents, the decisions they made concerning healthy weight in early childhood were also influenced by their ethnic identity. Equally, they cited their cultural beliefs as a motivation when discussing breastfeeding and weaning practices. The breastfeeding duration for all the women who participated in the FG ranged from 6-36 months. However, the men argued that they didn't have a role in supporting women to breastfeed:

Well, I don't have anything to do with that, it's the women's role and they manage that very well without us men. It's just 
not our culture to get involved with the breastfeeding, that's for the women. (Parents FG-3)

The HVs all encouraged breastfeeding for at least the first six months of a child's life, and every HV from the three FGs articulated the advantages of breastfeeding:

You can get very young children, and babies can be overfed easily from bottle-feeding whereas breastfeeding is not possible. The child will take what they need but in bottlefeeding, parents can sort of make them take a bit more. We encourage breastfeeding as a Trust. (HVs FG-2)

The majority of the HVs felt that they did not have enough knowledge of the African-centric diet to support parents with confidence. Therefore, this was identified as an area of training needed by HVs, with related comments including the following:

Most of the Black African families live in areas where its mainly white health visitors going to them who don't understand their food, doesn't understand what they eat, so it's difficult for them to really say something for them to understand. (HVs FG-3)

We have these booklets and we all have them and we can refer to them to help with our discussions with parents... we should have that for the African families too; that would really help us. (HVs' FG-1)

The role of family and friends

In every FG, the participants discussed the role of family and social ties, coming to the conclusion that both influenced their decisions about their child's diet and weight. Families and friends were relied on for advice on child-rearing, nutrition, and child development, with participants feeling they were an important source of knowledge: 
Actually, my friends who are living here in the UK who have got kids older than mine, I always ask them. Actually, I do ask them about food for my son... They do help me and we exchange experiences. But also, sometimes I think the problem with friends is that they would say that a child is healthy because it's chubby. Some of them will say, "how come your baby is so thin" so you try to feed your child more just because you want your child to look chubby. (Parents' FG-1)

\section{Influence of children}

Interestingly, there was an indication that children as young as two-years old played a key role in influencing their own dietary patterns. Some parents explained how they considered their children's eating habits, likes, and dislikes when deciding what to feed them:

My child is about two years of age. When he was younger I gave him what I thought was good for him, but now he's so fussy about food and I am kind of now led by him in a way. (Parent FG-1)

\section{Advice from health practitioners}

Although the participants were receptive to messages of a healthy diet, and although they valued the role of HVs in advising them about a healthy diet, they felt that the African heritage diet was not regarded, as indicated in a lack of consideration for their culture in the advice they received from health and social care professionals:

My son, he was only eating fufu every single day, so when I asked my health visitor she doesn't know anything about fufu. Since now there are so many different cultures health visitors and other health people should learn about different cultures have their meals. Probably not specific countries but, like, let's say in Africa what do you eat and advise us on our food. (Parents FG-2)

Parents also expressed some ambiguity about the advice of not adding salt to their children's diets. Confusion was expressed on this topic because 
some baby food bought from the shops always included salt, and in addition, they also felt it was impractical not to add salt as the child got older:

They (HVs) tell you no salt, no sugar, but I couldn't because to me, I have other children, my sisters, my brothers, we eat normal food with salt and normal sugar in moderation, not too much and nothing happens. So I didn't think it was really not possible because what I didn't want was for her [referring to her daughter] to not get used to having salt and then by the time she is older she may not like our food. And that's what I have at home, not too much salt but enough to make the food tasty. (Parents FG-2)

In general, the HVs felt that their training equipped them with the skills required in order to ensure the advice given to parents was consistent. However, some of them stated that although they were confident in providing the mainstream advice on healthy diet in childhood, they did not feel competent in addressing culturally specific support to Black African parents:

We work with all families, if we see a child that is overweight we call the Universal Plus and we have to do four contacts with them and do some work with them. Or, if they are underweight as well, we do the same thing so we have to see them. I mean, I usually do the first and then I give it to the nurse to follow up because I feel they would have more time to go home, look at what they are actually eating there, because we can't do that on a regular basis. So, they go home and they sit with them. But it's a cultural thing again it's very difficult to provide the culturally specific food all you can talk about is the portion sizes. (HVs' FG-3)

Despite the lack of culturally specific knowledge, the HVs in all three FGs articulated their role in supporting families during the early years of a child's life, with them believing they had an important role regarding the health and wellbeing of children in early childhood. They described what 
was involved with the key visits to families as well as the range of advice and support provided to those families.

\section{Challenges to healthy weight maintenance in early childhood}

While there was an overwhelming acceptance of the need for healthy weight maintenance in childhood, all participants from the parents FGs identified a number of key challenges that act as barriers for their community.

\section{Physical environment and socio-economic status}

The participants described how their environments had higher than average amounts of fast food, a lack of recreational facilities for children, and a lack of access to outlets that provided wholesome food. They also articulated how food accessibility and prices influenced the choices they made for their children's diet (more than health messages) for increasing consumption of healthy foods. In order to accommodate their realities which included working long hours and receiving low wages, there was a substantial dependence on ready-made meals for children.

Although all the parents preferred the African heritage diet, given their lived experiences they also relied on convenient and ready-made meals. This is because, as the participants noted, shopping for African heritage food was expensive and time-consuming in its preparation. Nonetheless, a view was expressed that it would be helpful if the African heritage food-stuff and diet was promoted and included in healthy diet advice and messages.

The discussions with HVs focused on the feasibility and effectiveness of an environmental intervention for improving diet by comparing the impact of health messages and lowered prices, as well as the two combined: 
Some of our parents are quite poor as well, you know, and if they are new to the country, they could be refugees so they've lost most things. Food costs would itself bar against a healthy diet, so it's not just giving messages but also looking at affordability too. (HVs' FG-3)

The takeaways are cheaper now ..., so our parents just have to be realistic when buying food. (HVs' FG-2)

\section{Language barriers, immigration status and discrimination}

Language barriers, immigration status, and discrimination were also identified as central factors that underpinned families' struggles for a healthy weight maintenance in early childhood. In all of the FGs there were discussions about how uncertainty in immigration status and discrimination would curtail their efforts to focus on their children's health. However, in their discussions it became clear that immigration status and experiences of social discrimination as an explanatory category were not easy to describe. Typical responses to the subject included:

When I came to this country, when I arrived at the airport, I was tested and they said my health was excellent, OK. Then, when I told them what I had been through, they were shocked, so while waiting for my status I was detained with my family and from detention, we were given a place to live, but the stress was just too much... You can't think of healthy diet when your immigration status is uncertain, you just can't think of anything else. It's important that all that is taken in account also, how the immigration status affect us. (Parents FG-3)

Some parents described the discrimination they encountered in employment which restricted their social mobility and ability to afford a healthy diet. These factors led to poor living conditions, which were a great source of stress and which undermined their family's mental wellbeing. The findings illustrate that Black African working-class families have additional 
factors and stresses that influence their efforts to promote a healthy weight in early childhood in comparison with white working-class families.

\section{An integrated approach to promote healthy weight in childhood}

The participants readily identified several factors that could facilitate the promotion of healthy weight maintenance in early childhood. It was accepted, for instance, that obesity in childhood does not occur in isolation, therefore any intervention should address a number of key areas, as identified below.

Training and guidance for HVs to provide culturally specific healthy eating advice

Given that participants discussed how HVs lacked the guidance needed to provide culturally specific advice to Black African parents, a significant step towards promoting healthy weight in childhood amongst the Black African community (which was identified across all seven FGs) was the need for specific training that prepares HVs to work with Black African parents:

It would be great to have culturally appropriate resources. $A$ lot of our families, they do like cassava and I don't even know what that is and that's what they ask me, is this OK to give our child, and I have to go and find out what it is and, you know, whether it's appropriate for their child to have it. So maybe a bit of culture learning for us as well. (HVs FG-1)

Culturally specific advice and practices related to food and the feeding of children emerged as a key need in all of the FGs, with the participants calling for culturally tailored interventions and an awareness of the lived experiences of the Black African community in the UK:

Having a cultural awareness in a way is important, or someone who has got that awareness to advise about our food. (Parents' FG-1) 
I think giving training to health visitors and others in the clinic to help provide culturally appropriate advice on diet for children would be good. It makes sense if they have the training, that would also help us. (HVs' FG-3)

\section{Practical resources and interactive guidance}

Most parents (and some HVs) also identified a need for practical resources and interactive guidance for parents, community organisations, and health and social care staff on how to more effectively engage and support families with the maintenance of a healthy weight in early childhood:

I've sometimes thought in my health clinic it would be great to have a lot more pictures up. Because if you say something to some of our migrant parents like with your child, you need to start ... for example, the response at times is, they're what? So pictures would be very helpful (HVs FG-2)

\section{A systems-based intervention}

Although participants in all seven FGs identified the need for a systembased intervention, including an awareness of families' social and economic influences, as well as strengthening local policies and media outlets to enable healthy weight maintenance in childhood:

Parents also need support with other things. It would be easier if access to healthy food was easier and if there were opportunities for good playing places for children... it's like you need the whole system to work for them, our work is important but in isolation it won't be helpful if parents are struggling with other things in their lives, which could also include immigration and jobs. (HVs FG-2).

\section{Discussion}

In this project, the Black African parents appeared to be well informed about the advantages of a healthy diet. However, the maintenance of a healthy weight and diet was only a contributing part of improving a child's 
wellbeing, with the parents arguing that their cultural values, identity, and socio-economic and migration status (including experiences of discrimination) were all important determinants of their children's nutritional status. This finding is similar to other studies, which have highlighted that complex factors beyond personal decisions strongly influence the maintenance of healthy weight and dietary choices and patterns (Mozaffarian et al., 2018; Ochieng 2011; Lavell, 2016).

The results also highlighted a common underlying need from the Black African parents for accurate and practical advice regarding different cultural diets, as well as the need for cultural perspectives on the messages given regarding healthy weight in early childhood. Unfortunately, their receptivity to advice from HVs and other health and social care providers was limited because it appeared to conflict with their lived experiences and at times their cultural beliefs, as well as acting as a barrier on messages regarding healthy diet and weight. This finding concurred with previous work in the US (Kumanyika 2008; McGary, 1999). The manifestations of uncertainty in immigration and settlement status, including experiences of discrimination, were manifold, and their impact limited the choices available for Black African parents and their children to maintain healthy weight in childhood. Several studies (Ochieng, 2013; Nazroo, 2001), have also identified the crippling effects of racism on the health and wellbeing of Black and minority ethnic families, the consequences of which were manifested in the social structure of most Black and other minority ethnic families.

Although there is limited empirical evidence that provides information on how experiences of uncertainty in immigration status and discrimination by parents can impact children's dietary requirements and wellbeing, a number of studies have highlighted the intricacies and subtlety of racism in individual's lives, and its impact on their health and wellbeing (Authors; 
Henry, 2010; Perkins and Repper, 2013). Other studies have also highlighted how uncertainty in migration status could limit an individual's capacity to participate in a healthy diet and lifestyle (Zilanawala, 2015). In this study, parents described their frustration at how these factors undermined their mental wellbeing and socio-economic status. All of this resulted in an inability to provide appropriate care for their children, the resultant labour inequality and other structural barriers attributing to their children's poor health and wellbeing. In the US, a number of authors (Björntorp, 2001; Dallman et al., 2003; Adam and Epel, 2007) described how the day-to-day stress caused by a socio-political history of oppression amongst some African Americans resulted in reliance on several coping strategies, which in turn resulted in being overweight and suffering from obesity.

Although the influences on the predisposition to childhood obesity suggest the need for culturally tailored interventions (Wang and Tussing, 2004; Daniels et al., 2005), consideration must also be given to the relevant social, economic, local, and national policy (Booth et al., 2001; Wetter et al, 2001; Satia-About, 2002). Critically, a number of factors that emerged from this project have also been identified by other minority groups as well as low socio-economic families from the majority population in the UK as a whole, in particular the influence of socio-economic status (PHE, 2018 \& 2015). This suggests that interventions should be designed to be robust enough to be specific to Black African communities while also being able to reach out to all communities. Such "mainstream" interventions could address many of the identified factors such as food pricing, deprivation, and cultural and social-specific messages, while also including strategies to address challenges such as how health and social care practitioners respond to the health problems related to immigration status, and the lived experiences of migrant families (Flynn, 2006; Warschburger \& Kroeller, 
2012). It is also recommended for health and social care providers to engage with communities, for local authorities to develop a partnership model that could address the massive inequality in accessing a healthy diet, to limit the proliferation of unhealthy food outlets in inner city areas.

Similar to the work of Redsell (2013), the HVs in this project felt they had neither the skills nor the guidance to have discussions regarding healthy weight maintenance in early childhood with Black African parents. Equally, the parents who participated in the project also advocated for training to enable HVs and other health and social care providers to provide culturally appropriate interventions. However, as argued by Page (2005), cultural influences evolve and respond to environmental pressures and context and, therefore, in designing training it is important not only to focus on cultural attitudes and beliefs but also to provide guidance on the historical and socio-political circumstances and lived experiences of migrant communities that support the cultural variables.

An analysis of the Infant Risk of Obesity Checklist (Weng et al., 2013a) and The Guidelines for Public Health Nurses - UK health visitors (Redsell et al., $2016 ; 2013 a \& b$ ) illustrate that both tools provide interactive intervention strategies, including guidance for UK HVs on how to help facilitate discussions about obesity prevention with parents during routine home visits. However, upon review, the tools do not appear to take into account the lived experiences of Black and other minority ethnic communities, and their application has also resulted in mixed findings. As the results of this project have highlighted, environmental influences, combined with sociocultural and economic status on behaviour, need to be integrated into strategies that promote healthy weight maintenance in early childhood. 
Consequently, a system-based strategy that promotes interrelationships between the factors identified in this project is important if we are to promote healthy weight in early childhood in Black African children. Such a tool should also be robust enough so that it can be customised to meet the particular needs of different communities. At the family level, the intervention could include healthy weight maintenance based on nutrition education and the enhancement of physical activity, while at the macro level it would need the support of the local authorities, Clinical Commission Groups, the food industry, and the mass media in order to achieve and sustain changes, making sure the healthier options are also easy options.

This study was designed to provide a community understanding of healthy weight maintenance, and the use of FGs enabled in-depth discussions with the Black African migrant parents that more completely covered their understanding and perception of healthy weight maintenance in early childhood. A potential limitation of this project is that the focus was not on parents of children with poor weight maintenance, such as: overweight, obese, or underweight. Future studies could focus on parents' experiences of accessing services that promote healthy weight strategies in early childhood. Findings from such a study could then add to the emerging literature on weight management strategies for children.

\section{References}

Adam, T. and Epel, ES. (2007). Stress, eating and the reward system. Physiology and Behaviour Journal, 9(1), 449-58.

Baldwin, S (2012) Exploring the professional identity of health visitors. Nursing Times, 108, 12-15.

Bjöntorp, P. (2001). Do stress reactions cause abdominal obesity and comorbidities? Obesity Review, 2, 73-86. 
Blüher, M. (2019) Obesity: global epidemiology and pathogenesis. Nature Reviews Endocrinology, 15, 288-298.

Booth, SL., Sallis, JF., Ritenbaugh, C., Hill, JO., Birch, LL., Frank, LD. (2001) Environmental and societal factors affect food choice and physical activity: rationale, influences, and leverage points. Nutrition Reviews, 59, S21-39.

Bryman, A. (2016) Social Research Methods. Oxford: Oxford University Press.

Census (2011) https://www.ons.gov.uk/census/2011census (accessed 4th October 2017)

Cheng, TO. (2012). Childhood obesity in modern China. International Journal of Cardiology, 157, 315-317.

Dallman, MF., Pecoraro, N., Akana, SF., La Fleur, SE., Gomez, F. and Houshyar H. (2003). Chronic stress and obesity: a new view of "comfort food". Proceedings of the National Academy of Sciences of USA, 100, 11696-11701

Daniels, SR., Arnett, DK., Eckel, RH., Gidding, SS., Hayman, LL. and Kumanyika, S. (2005). Overweight in children and adolescents: pathophysiology, consequences, prevention, and treatment. Circulation, 111, 1999-2012.

Dawe, N. (2015). Addressing childhood obesity: Overcoming the barriers. British Journal of School Nursing, 10(9), 447-450.

Department of Health (DH). (2009). Healthy weight, healthy lives: a cross government strategy for England. London: Department of Health.

Department of Health (2012). Getting it right for children, young people and families: Maximising the contribution of the school nursing team: Vision and Call to Action. London: The Stationery Office.

Dyck, ND., D'Haese S., Plaete J., Bourdeaudhuii DI., Deforche B. and Cardon. (2019) Opinions towards physical activity interventions using Facebook or text messaging: Focus group interviews with vocational school-aged adolescents, Health and Social Care in the Community, 27, 654-664.

Flynn, MA., McNeil, DA., Maloff, B., Mutasingwa, D., Wu, M., Ford, C. and Tough, SC. (2006). Reducing obesity and related chronic disease risk in 
children and youth: a synthesis of evidence with 'best practice' recommendations. Obesity Reviews, 7(1), 7-66.

Gatineau, M. and Mathrani, S. (2011). Ethnicity and obesity in the UK. Perspectives in Public Health, 131(4), 159-160.

Golley, RK., Magarey, AM., Baur, LA., Steinbeck, KS. and Daniels, LA. (2007). Twelve-month effectiveness of a parent-led, family-focused weight-management program for prepubertal children: A randomized, controlled trial. Pediatrics, 119, 517-525.

Guo, SS., and Chumlea, W. (1999). Tracking of body mass index in children in relation to overweight in adulthood. The American Journal of Clinical Nutrition, 70(1), 145S-148S.

Health and Social Care Information Centre (HSCIC). (2018). Statistics on Obesity, Physical Activity and Diet [online]. Available at https://digital.nhs.uk/data-andinformation/publications/statistical/statistics-on-obesity-physical-activityand-diet/statistics-on-obesity-physical-activity-and-diet-england-2018 [accessed 20 June 2018].

Henry, W. (2010). Conceptualisation and effects of social exclusion, racism and discrimination and coping strategies of individuals and families in Ochieng BMN. and Hylton CLA., Black Families in Britain as the Site of Struggle. Manchester, UK: Manchester University Press, pg 105-125.

House of Commons (HC) Health Committee. (2018). Childhood obesity: Time for action: Eighth Report of Session 2017-19. London: House of Commons.

Hughes $L$ (2016) More spent on treating obesity-related conditions than on the police or fire service, says NHS Chief. The Telegraphy, $7^{\text {th }}$ June. https://www.telegraph.co.uk/news/2016/06/07/more-spent-ontreating-obesity-related-conditions-than-on-the-po/ (Accessed $18^{\text {th }}$ April 2019)

Kelishadi, R. and Azizi-Soleiman, F. (2014). Controlling childhood obesity: A systematic review on strategies and challenges. Journal of Research in Medical Sciences, 19, 993-1008

Kvaavik, E., Tell, GS. and Klepp, KI. (2003) Predictors and tracking of body mass index from adolescence into adulthood. Follow-up of 18 to 20 years 
in the Oslo Youth Study. Archives of Pediatric and Adolescent Medicine, $157,1212-1218$.

Kumanyika, SK. (2008) Environmental influences on childhood obesity: Ethnic and cultural influences in context. Physiology and Behaviour, 94, $61-70$

Lacey, A and Loft, D. (2009)Qualitative Data Analysis https://www.rdsyh.nihr.ac.uk/wpcontent/uploads/2013/05/9 Qualitative Data Analysis Revision 2009.pd f (Accessed 16th June 2018)

Lavelle, F., McGowan, L., Spence, M., Caraher, M., Raats, MM., Hollywood, L., McDowell, D., McCloat, A., Mooney, E. and Dean, M. (2016). Barriers and facilitators to cooking from 'scratch' using basic or raw ingredients: A qualitative interview study. Appetite, 107, 383-391

Maynard, MJ., Baker, G., Rawlins, E., Anderson, A. and Harding, S. (2009). Developing obesity prevention interventions among minority ethnic children in schools and places of worship: The DEAL (DiEt and Active Living) study BMC Public Health, 9, 480 doi: https://doi.org/10.1186/1471-2458-9-480

McGary, H. (1999). Distrust, social justice, and health care. Mount Sinai Journal of Medicine, 66, 236-40.

Mozaffarian, D., Angell, SY., Lang, T. and Rivera, JA. (2018). Role of government policy in nutrition-barriers to and opportunities for healthier eating, BMJ, 361, doi: https://doi.org/10.1136/bmj.k2426

Nazroo, JY. (2001). Ethnicity, class and health. London: PSI.

NHS Health and Social Care (NHSC) Information Centre (2013). Statistics on obesity, physical activity and diet: England. Available at www.ic.nhs.uk. [Accessed 13 November 2018].

Ochieng BMN. (2013) Black families' perceptions of barriers to the practice of a healthy lifestyle: a qualitative study in the UK. Critical Public Health, 23(1): 6-16.

Ochieng BMN. (2011) Factors influencing the diet patterns and uptake of physical activity among Black families. International Journal of Health Promotion and Education, 49: 140-145. 
Ochieng BMN. (2010) "You know what I mean:" The ethical and methodological dilemma and challenges for Black researchers interviewing Black families. Qualitative Health Research, 20(12): 1725-1735.

Page, JB. (2005). The concept of culture: a core issue in health disparities. Journal of Urban Health, 82(3), iii35-iii43.

Perkins, R. and Repper, J. (2013). Prejudice, discrimination and social exclusion: reducing the barriers to recovery for people diagnosed with mental health problems in the UK. Neuropsychiatry, 3(4), 377-384.

Public Health England. (PHE) (2019) Differences in child obesity by ethnic group: Obesity prevalence data from the National Child Measurement Programme (NCMP) showing variations by ethnicity.

https://www.gov.uk/government/publications/differences-in-childobesity-by-ethnic-group/differences-in-child-obesity-by-ethnicgroup[Accessed 23 January 2019].

Public Health England (PHE). (2018). The Healthy Child Programme Best start in life and beyond: Improving public health outcomes for children, young people and families Guidance to support the commissioning of the Healthy Child Programme 0-19: Health visiting and school nursing services (commissioning guide 1, 2 \&3). London: PHE.

Public Health England (PHE). (2016) The Eatwell Guide. https://www.gov.uk/government/publications/the-eatwell-guide (Accessed 20 August 2018)

Public Health England (PHE). (2015). Childhood obesity: applying all our Health. London PHE.

Redsell, S., Swift, JA. and Nathan, D. (2013). UK health visitors' role in identifying and intervening with infants at risk of developing obesity. Maternal and Child Nutrition, 9, 396-408.

Satia-About, AJ., Patterson, RE., Neuhouser, ML. and Elder, J. (2002). Dietary acculturation: applications to nutrition research and dietetics. Journal of the American Dietetic Association, 202(8), 1105-1118.

Simmonds, M., Burch, J., Llewellyn, A., Griffiths, C., Yang, H. and Owen C. (2015) The use of measures of obesity in childhood for predicting obesity and the development of obesity-related diseases in adulthood: a systematic review and meta-analysis. Health Technology Assessment, 19(43). ISSN 1366-5278. 
Wang, Y. and Tussing, L. (2004). Culturally appropriate approaches are needed to reduce ethnic disparity in childhood obesity. Journal of the Academy of Nutrition and Dietetics, 104, 1664-1666.

Wetter, AC., Goldberg, JP., King, AC, Sigman-Grant, M., Baer, R. and Crayton, E. (2001) How and why do individuals make food and physical activity choices? Nutrition Review, 59, 11-20.

World Health Organization (WHO). (2016). Report of the commission on ending childhood obesity. Geneva: WHO

Zilanawala, A., Davis-Kean, P., Nazroo, J., Sacker, A., Simonton, S. and Kelly, Y. (2015) Race/ethnic disparities in early childhood BMI, obesity and overweight in the United Kingdom and United States, International Journal of Obesity, 39, 520-529. 